\title{
Genome-wide identification and expression analysis of the $\beta$-amylase genes strongly associated with fruit development, ripening, and abiotic stress response in two banana cultivars
}

\author{
Hongxia MIAO ${ }^{1^{*}}$, Peiguang SUN ${ }^{2 *}$, Yulu MIAO ${ }^{3 *}$, Juhua LIU ${ }^{1}$, Jianbin ZHANG ${ }^{1}$, Caihong JIA ${ }^{1}$, Jingyi WANG ${ }^{1}$, \\ Zhuo WANG', Zhiqiang JIN $(\bowtie)^{1,2}$, Biyu XU (凶) ${ }^{1}$ \\ 1 Key Laboratory of Tropical Crop Biotechnology, Ministry of Agriculture, Institute of Tropical Bioscience and Biotechnology, \\ Chinese Academy of Tropical Agricultural Sciences, Haikou 571101, China \\ 2 Key Laboratory of Genetic Improvement of Bananas, Hainan Province, Haikou Experimental Station, \\ Chinese Academy of Tropical Agricultural Sciences, Haikou 570102, China \\ 3 Department of Agriculture, Hainan University, Haikou 570228, China
}

\begin{abstract}
BAM) is an important enzyme involved in conversion of starch to maltose in multiple biological processes in plants. However, there is currently insufficient information on the BAM gene family in the important fruit crop banana. This study identified $16 B A M$ genes in the banana genome. Phylogenetic analysis showed that MaBAMs were classified into four subfamilies. Most MaBAMs in each subfamily shared similar gene structures. Conserved motif analysis showed that all identified MaBAM proteins had the typical glyco hydro 14 domains. Comprehensive transcriptomic analysis of two banana genotypes revealed the expression patterns of $M a B A M s$ in different tissues, at various stages of fruit development and ripening, and in responses to abiotic stresses. Most MaBAMs showed strong transcript accumulation changes during fruit development and late-stage ripening. Some MaBAMs showed significant changes under cold, salt, and osmotic stresses. This finding indicated that MaBAMs might be involved in regulating fruit development, ripening, and responses to abiotic stress. Analysis of five hormone-related and seven stressrelevant elements in the promoters of MaBAMs further revealed that BAMs participated in various biological processes. This systemic analysis provides new insights into the transcriptional characteristics of the $B A M$ genes in banana and may serve as a basis for further functional studies of such genes.
\end{abstract}

Keywords $\beta$-amylase, banana, genome-wide identification, fruit development, abiotic stress

Received November 1, 2016; accepted December 15, 2016

Correspondences: 18689846976@163.com, biyuxu@126.com

${ }^{*}$ These authors contribute equally to the work

\section{Introduction}

Starch is the main storage carbohydrate in plants, and is rapidly degraded to release the primary chemical energy and organic matter for grain germination, seedling growth, endosperm development, and response to abiotic stresses through five major enzymes, including $\alpha$-amylase (AMY), $\beta$-amylase (BAM), limit dextrinase (PUL), $\beta$-glucosidase, and $\alpha$-glucan phosphorylase $(\mathrm{PHO})^{[1-5]}$. AMY initiates starch breakdown in the germinating grain ${ }^{[6]}$. BAM converts starch to $\beta$-maltose $\mathrm{e}^{[7,8]}$. PUL catalyzes the hydrolysis of $\alpha-1,6$ glucosidic linkages to release dextrins ${ }^{[9]}$. $\beta$-glucosidase is responsible for efficient hydrolytic activity ${ }^{[10]}$. PHO is mainly involved in the reversible cleavage of $\alpha$-1,4-linked glucose polymers into $\alpha$-Dglucose-1-phosphate ${ }^{[11]}$. However, several studies found that AMY, PUL, $\beta$-glucosidase, and PHO only have minor roles in transient starch breakdown in living tissues (e.g., Arabidopsis thaliana leaves) ${ }^{[12,13]}$, while BAM is the major enzyme ${ }^{[14]}$

BAM catalyzes the hydrolysis of $\alpha-1,4$ linked oligo and poly glucans and is primarily responsible for hydrolysis of starch and degradation of transient starch to generate $\beta$ maltose as the predominant sugar exported from the chloroplast at night ${ }^{[8,15]}$. BAM is a member of glycosyl hydrolase family 14 . It has typical glyco hydro 14 domains (PF01373) at the $\mathrm{N}$ terminus and a relatively central localization, is widely distributed in higher plants and microorganism and is encoded by a multigene family ${ }^{[7,8,15]}$. So far, genome-wide analyses have identified nine BAMs in Arabidopsis ${ }^{[5]}, 10$ in rice, 13 in corn, 11 in Brachypodium distachyon, 10 in sorghum, and 10 in millet ${ }^{[16]}$. Biochemical and genetic analyses have revealed that the function of $B A M$ genes is related to plant 
germination, growth, development, and maturation. A BAM protein from fenugreek was reported to participate in seed germination ${ }^{[15]}$. Overexpression of $B A M 7$ and $B A M 8$ in Arabidopsis controlled shoot growth and development through crosstalk with brassinosteroid signaling ${ }^{[17]}$. Expression of Bmyl gene in endosperm was involved in the development and maturation of barley grain ${ }^{[18]}$. In addition, expression analyses of the $B A M$ gene family in various species indicated that its expression is altered in response to various abiotic stresses, such as cold, salt or drought ${ }^{[19-21]}$. Further studies support the suggestion that $B A M$ genes play a role in plant response to abiotic stress. Mutant analysis indicated that Arabidopsis BAM1 protein decreases stomatal opening and improves drought tolerance, due to reduced starch breakdown in guard cells ${ }^{[20]}$. Arabidopsis BAM8 has been found to be involved in cold tolerance and maltose accumulation ${ }^{[22]}$ while BAM proteins in barley has been identified as important regulators of salt tolerance ${ }^{[23]}$. Taken together, these studies have shown that the $B A M$ gene family is involved in regulating plant germination, growth, development, maturation, and response to various stresses.

Banana (Musa acuminata) is not only the most popular high-starch fresh fruit (almost 20\% in unripe banana fruit) but also an important staple food in some Africa and Latin American countries ${ }^{[24,25]}$. Starch degradation to sugar is very important in banana, since sugar concentration is a major component of fruit quality and economic value. Meanwhile as a large annual monocotyledonous herbaceous plant, banana production is frequently destroyed by various abiotic stresses during growth and development, such as cold, salt and drought ${ }^{[26-28]}$. Thus, investigations of key candidate genes involved in fruit development and responses to multiple stresses, based on complete genome sequences, are necessary to improve quality and enhance stresses tolerance ${ }^{[29,30]}$. However, few studies on identification and characterization of banana genes based on genome sequencing data have been reported ${ }^{[30]}$. To our knowledge, only one $B A M$ gene has been reported to have been cloned in banana ${ }^{[31]}$. Considering the importance of the $B A M$ gene family in plant growth and development, as well as response to abiotic stresses, we decided to conduct a genome-wide analysis of the banana genome.

In this study, we identified $16 M a B A M$ genes and analyzed their phylogenetic relationship, gene structure, protein motifs, and expression patterns in different tissues, different stages of fruit development and ripening, and the response to abiotic stresses (cold, salt, and drought) in two banana genotypes. Further, we analyzed the hormonerelated and stress-relevant cis-elements in the promoters of $M a B A M$ genes. This comprehensive study has increased our understanding of $B A M$ genes associated with fruit development processes and abiotic stresses responses, and will provide a crucial foundation for future studies of crop improvement medicated by BAMs.

\section{Materials and methods}

\subsection{Plant materials and treatments}

In this study, two banana cultivars, BaXi Jiao (M. acuminata AAA group cv. Cavendish, BX) and Fen Jiao ( $M$. AAB group Fenjiao, FJ), were selected to perform comparative analyses. $\mathrm{BX}$ is a triploid cultivar with high yield, high quality, and long-term storage, and is widely cultivated in tropical and subtropical regions in China. FJ is a triploid cultivar, but with a different genotype (AAB) compared with BX (AAA), and has the characteristics of good flavor, rapid ripening, and tolerance to abiotic stresses. It is widely cultivated in the Guangdong and Hainan Provinces of China. The two cultivars were obtained from the banana plantation at the Chinese Academy of Tropical Agricultural Sciences (Danzhou, Hainan, China). Roots, leaves, and fruits at 80 days after emergence from the pseudostem (DAF) were sampled for expression analysis in different tissues. For the banana fruit developmental process, fruits of 0,20 , and $80 \mathrm{DAF}$, which represent fruit developmental stages of budding, cutting flower, and harvest stages, were collected from both cultivars. For postharvest ripening, BX fruit were stored for 0,8 , and $14 \mathrm{~d}$ postharvest (DPH) and FJ 0,3 , and $6 \mathrm{DPH}$, which represented ripening stages of green, more green than yellow and full yellow according to Pua et al. ${ }^{[32]}$. Five-leaf stage young banana plants of both cultivars with uniform growth were grown in soil at $28^{\circ} \mathrm{C}, 70 \% \mathrm{RH}$ and $200 \mu \mathrm{mol} \cdot \mathrm{m}^{-2} \cdot \mathrm{s}^{-1}$ light intensity in $16 \mathrm{~h} \mathrm{light} / 8 \mathrm{~h}$ dark cycle. For salt and osmotic treatments, five-leaf stage banana plants were irrigated with $300 \mathrm{mmol} \cdot \mathrm{L}^{-1} \mathrm{NaCl}$ and $200 \mathrm{mmol} \cdot \mathrm{L}^{-1}$ mannitol for $7 \mathrm{~d}$. For cold treatment, banana plants were maintained at $4^{\circ} \mathrm{C}$ for $22 \mathrm{~h}$.

2.2 Identification and phylogenetic analyses of the MaBAM gene family in banana

Whole banana (M. acuminata) protein sequences were downloaded from the banana genome database (http:// banana-genome.cirad.fr) ${ }^{[25]}$. BAM amino acid sequences from Arabidopsis and rice were obtained from the TAIR (http://www.arabidopsis.org) and RGAP (http://rice.plantbiology.msu.edu) databases, respectively. The Hidden Markov Model (HMM) profiles of the BAM typical conserved domains (glyco hydro 14 domains; PFAM: PF01373) (http://pfam.sanger.ac.uk) were used to query the predicted BAM proteins in the banana genome databases using HMMER software (http://hmmer.org) ${ }^{[33]}$. BLAST analysis was also used to identify the predicted banana BAMs with all BAMs from Arabidopsis and rice as queries. Conserved domain search of the potential banana BAMs was further validated using CDD (http://www.ncbi. nlm.nih.gov/cdd) and PFAM (http://pfam.sanger.ac.uk) databases. The accession number of all identified banana 
BAMs are given in Table S1 (Appendix A). Phylogenetic analysis of BAM amino acid sequences from banana, Arabidopsis, and rice was carried out using Clustal X 2.0 and MEGA 5.0 software with bootstrap values for 1000 replicates ${ }^{[34]}$.

\subsection{Protein properties and gene structure analysis}

Molecular mass and isoelectric points of the MaBAM proteins were predicted using the ExPASy database (http:// expasy.org). Motifs of MaBAM proteins were analyzed using MEME software (http://meme-suite.org) and annotated by InterProScan database search (http://www.ebi.ac. uk/Tools/pfa/iprscan). Structural features of the MaBAM genes were identified using Gene Structure Display Server (GSDS) software (http://gsds.cbi.pku.edu.cn). The sequences of promoters of $M a B A M$ genes were obtained from the banana genome database (http://banana-genome. cirad.fr $)^{[25]}$. The $2000 \mathrm{bp}$ upstream genomic sequences of these MaBAM genes was truncated to confirm the transcription start site using database (http://www.fruitfly. org/seq_tools/promoter.html) and the cis-acting elements via PlantCARE software (http://bioinformatics.psb.ugent. be/webtools/plantcare/html).

\subsection{Transcriptomic analysis}

Total RNA from each sample was extracted using a plant RNA extraction kit (Tiangen, China) and used to construct cDNA libraries according to the protocols supplied by Illumina. The sequencing was performed with an Illumina GAII following the manufacturer's instructions with two replicates per sample. The sequencing depth was 5.34X on average. Adapter sequences in the raw sequence reads and low quality sequences were removed using FASTX-toolkit and FastQC, respectively, and clean reads were obtained and mapped to the DH-Pahang genome (M. acuminata, $2 n=22$, A genome) ${ }^{[25]}$. Transcriptome assemblies were constructed with Cufflinks ${ }^{[35]}$. Gene expression levels were calculated as fragments per kilobase of exon per million fragments mapped (FPKM). DEGseq was used to identify differentially expressed genes.

\section{Results}

3.1 Identification and phylogenetic analysis of banana BAM family members

To identify all banana BAM family members, HMM searches using the BAM typical conserved domains (glyco hydro 14 domain; PFAM: PF01373) as queries, as well as BLAST searches using Arabidopsis and rice BAM sequences as queries, were performed in the banana genome database. After validating the BAM domain using the CDD and PFAM database, a total of 16 non-redundant $M a B A M$ genes were identified. The MaBAM genes were named according to their homologous genes in A. thaliana. The 16 predicted MaBAM proteins ranged from 224 (MaBAM5a) to 702 (MaBAM7a) amino acid residues and the relative molecular mass varied from 25.676 (MaBAM5a) to 77.438 (MaBAM7a) $\mathrm{kDa}$, with isoelectric points in the range of 5.00-9.56 (Appendix A, Table S1), suggesting their potentially different roles in regulating biological processes under different conditions.

To study the evolutionary relationships of BAM family proteins, a phylogenetic tree was constructed by aligning 9 , 10, and 16 BAM proteins from Arabidopsis, rice, and banana using Clustal X 2.0 and MEGA 5.0 software (Fig. 1). Based on the phylogenetic tree, all MaBAM proteins were grouped into four clades, Clades I, II, III, and IV. Clades I, II, and III are large with more than four MaBAM members, whereas Clade IV contains two MaBAM proteins, similar to those in the rice ${ }^{[5,16]}$. However, in banana, the number of MaBAM3 proteins in Clade I and MaBAM5 proteins in Clade II were obviously greater than in Arabidopsis and rice.

\subsection{Gene structure and conserved motifs analysis of banana $M a B A M$ genes}

The exon-intron structural evolution of the $M a B A M$ genes was mapped using the GSDS software. Evolutionary analysis further confirmed the classification of the 16 banana MaBAMs into four groups (Fig. 2), which was consistent with the cluster analysis (Fig. 1). Gene structure analysis showed that the MaBAM genes contained four exons in Clade I, three to seven exons in Clade II, eight to nine exons in Clade III, and three exons in Clade IV. This suggests that the majority of MaBAM genes in the same group share similar exon-intron organization and the gene structure might reflect gene evolution.

To explore the conserved motifs and functional prediction from banana MaBAM sequences, a total of 15 conserved motifs in the MaBAMs were identified using MEME software and annotated with the InterPro database (Fig. 3). The results suggest that 11 motifs (motifs 1-7 and motifs 9-12) were annotated as glyco hydro 14 domains, which are basic characteristics of the BAM gene family. All 16 identified MaBAMs contained the typical glyco hydro 14 domain motifs. All MaBAMs in Clade I had the motifs 1-13. Most MaBAMs (except MaBAM5a) in Clade II shared motifs $1-5,7-9,11-13$, and motif 14 at the C-terminal, whereas the MaBAMs in Clade III had motifs $1-7,9-13,15$ and lacked motif 14 at the C-terminal. MaBAM9a and MaBAM9b in Clade IV contained motifs $2-11,13$ and lacked motifs 1,12 , and 14-15. These results suggest that most MaBAMs in the same group share conserved motif regions and further support the phylogenetic analyses of banana BAMs. 


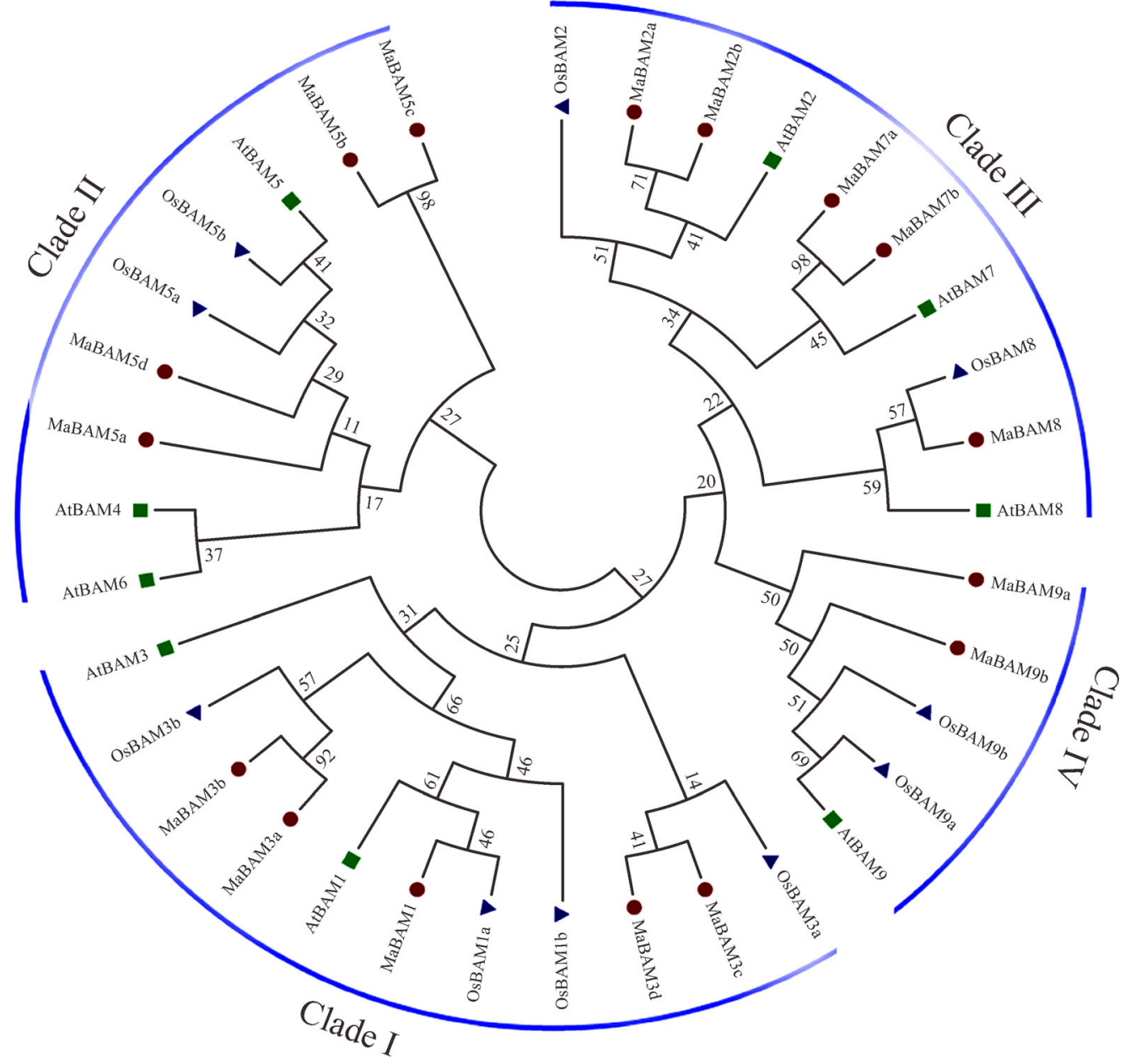

Fig. 1 Phylogenetic analysis of the BAMs from Arabidopsis, rice, and banana. The neighbor-joining (NJ) tree was drawn using MEGA5.0 with 1000 bootstrap. Four groups were shown as Clade I, II, III, and IV.

3.3 Expression patterns of MaBAM genes in different tissues of two banana cultivars

To investigate the role of $M a B A M$ genes in banana growth and development, expression patterns of $M a B A M$ genes in different tissues, including roots, leaves, and fruits, were tested in the two cultivars. Among the $16 M a B A M$ genes, 10 genes (excluding MaBAM2a, MaBAM3a, MaBAM3b, $M a B A M 5 c, M a B A M 5 b$, and MaBAM7b) showed expression in at least one tested tissue of two cultivars (Fig. 4; Appendix A, Table S2).

For BX, 9 (64\%), 9 (64\%), and 7 (50\%) MaBAMs were expressed in roots, leaves and fruits, respectively, among which $3(33 \%), 6(67 \%)$, and $2(29 \%)$ genes showed high expression levels $(>10)$ in roots, leaves, and fruits, respectively. Additionally, MaBAM9b exhibited high transcript levels $(>10)$ in all tissues examined.

For FJ, 8 (57\%), 8 (57\%), and 7 (50\%) MaBAM genes were expressed in roots, leaves, and fruits, respectively, among which $5(63 \%), 6(75 \%)$, and $3(43 \%)$ genes showed high expression levels $(>10)$ in roots, leaves, and fruits, respectively. Furthermore, three MaBAMs (MaBAM8, MaBAM9a, and MaBAM9b) had high expression levels $(>10)$ in all organs tested.

Comparison of the tissue expression profiles of $\mathrm{BX}$ and FJ revealed that some $M a B A M$ genes have similar expression patterns, including MaBAM1, MaBAM3c, $M a B A M 3 d$, MaBAM7a, and MaBAM9b. This indicates that these genes have similar functions in regulating tissues development in both cultivars. However, some genes exhibited differential expression patterns, e.g., MaBAM8 had abundant transcripts $(>17.6)$ in roots and fruits of FJ, but low transcripts $(<5.8)$ in roots and fruits of BX. MaBAM9a showed high expression levels $(>41.5)$ in FJ roots, but low expression levels $(<7.8)$ were detected in $\mathrm{BX}$ roots. This indicates that some MaBAM members have different roles during tissue development in the two cultivars. Additionally, the $M a B A M 9 b$ gene showed high expression levels $(>10)$ in all of the tested organs in both $\mathrm{BX}$ and FJ tissues, indicating a key role for the gene in 


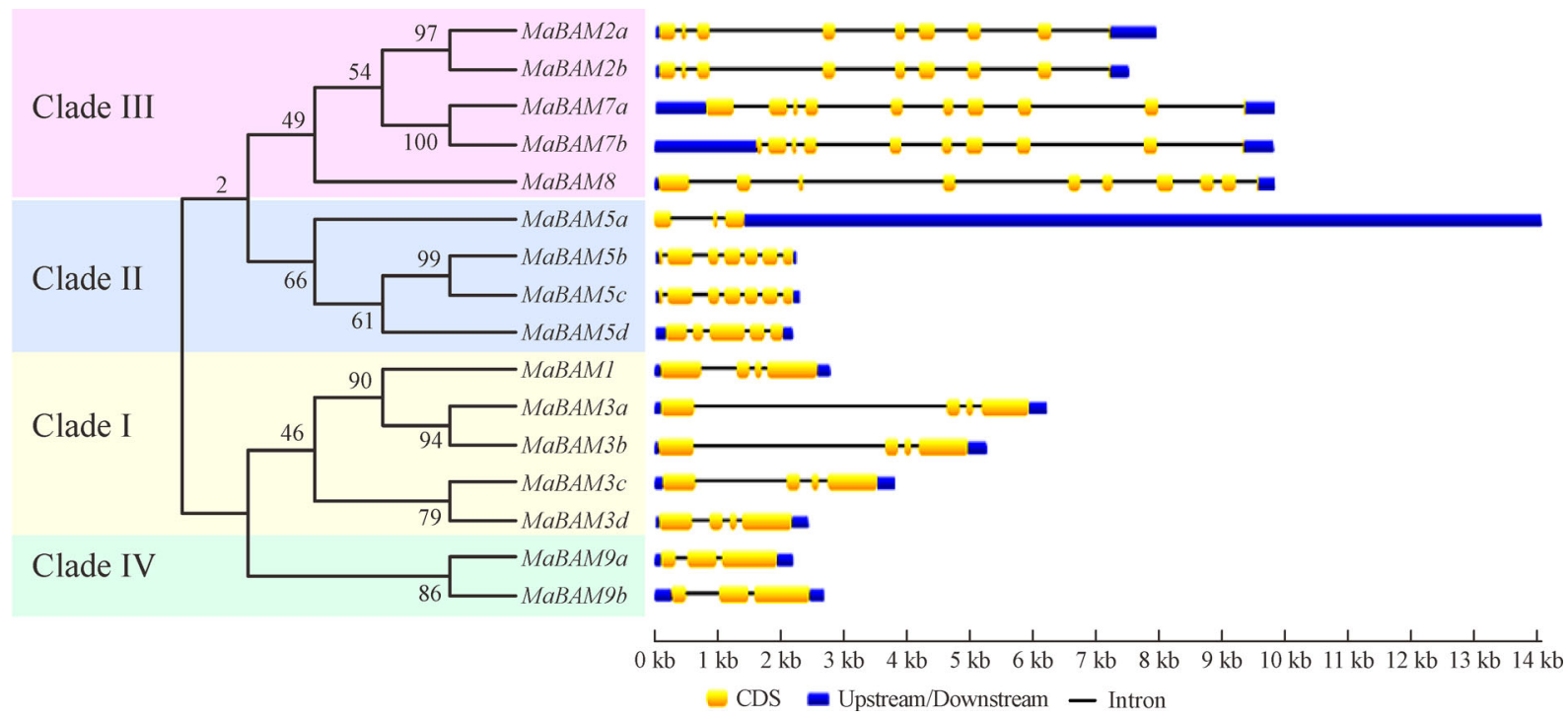

Fig. 2 Gene structure analyses of MaBAMs. Exon-intron structure analyses were performed by GSDS database. The blue boxes indicate upstream/downstream regions, the yellow boxes indicate exons, and the black lines indicate introns.

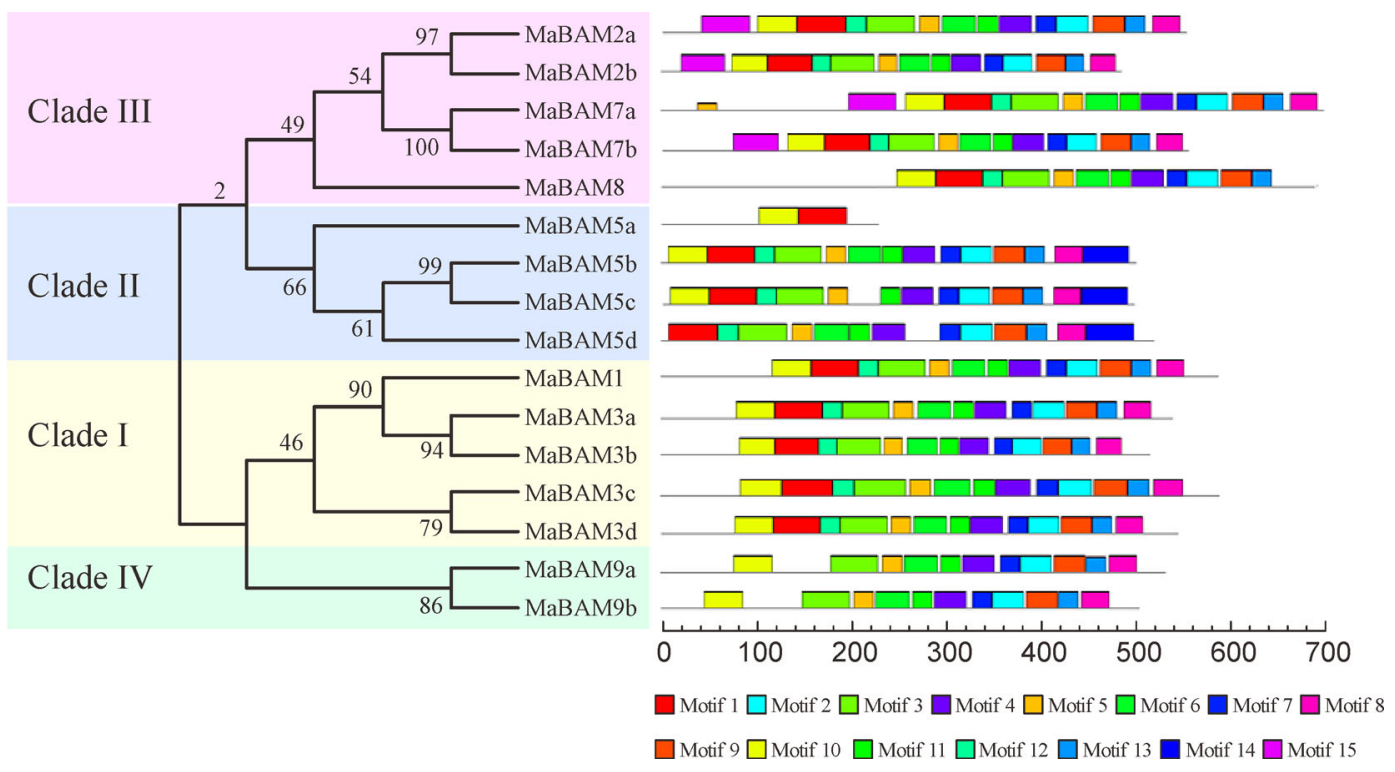

Fig. 3 Phylogenetic and motif analyses of MaBAMs. All motifs were identified by MEME database using the complete amino acid sequences of MaBAMs. The classification of MaBAMs was shown as Clade I, II, III, and IV based on the phylogenetic relationship.

regulating development of different tissues. Together, these tissue expression patterns of the $M a B A M$ genes in the two cultivars may provide insights for further study of tissue development and function.

3.4 Expression profiles of MaBAM genes in different stages of fruit development in two banana cultivars

To investigate the transcriptional response of MaBAM genes in banana during fruit development, the expression patterns of the MaBAM genes were analyzed in fruits sampled at 0, 20 and 80 DAF (Fig. 5; Appendix A,
Table S3). Among the 16 MaBAMs, 10 genes showed expression in different stages of banana fruit development.

For BX, 10, nine (no MaBAM5), and seven (no $M a B A M 2 a, M a B A M 3 d$, and MaBAM5a) MaBAM genes were expressed at 0,20 , and $80 \mathrm{DAF}$, with four (MaBAM3c, MaBAM8, MaBAM9a, and MaBAM9b), four (MaBAM3c, MaBAM8, MaBAM9a, and MaBAM9b), and two (MaBAM9a and MaBAM9b) genes, respectively, showing high expression levels $(>10)$ at 0,20 , and 80 DAF. Notably, MaBAM9a and MaBAM9b had high transcript accumulation $(>30)$ at all stages of fruit development. 


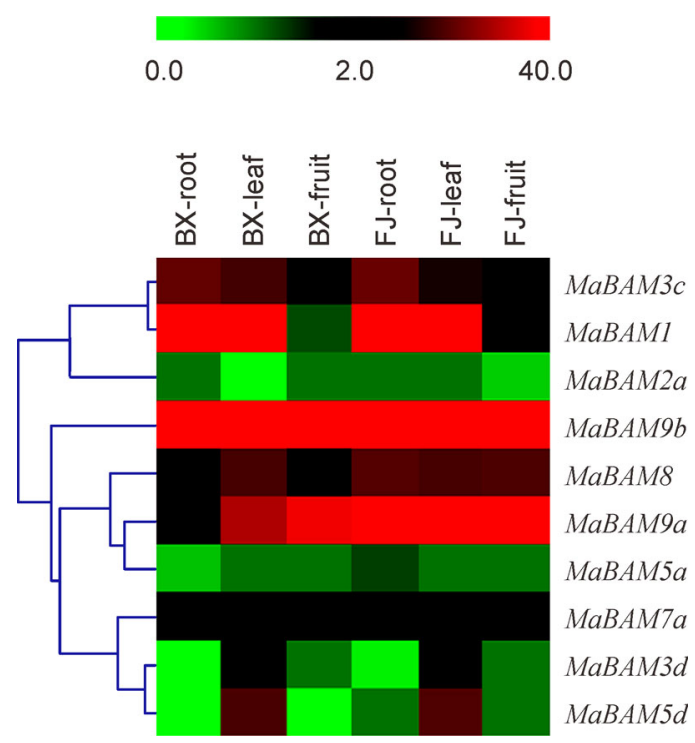

Fig. 4 Expression patterns of MaBAMs in roots, leaves, and fruits of two banana cultivars, BaXi Jiao (BX) and Fen Jiao (FJ). The heat map with clustering was created based on the fragments per kilobase of exon per million fragments mapped (FPKM) of MaBAMs. Differences in gene expression changes are shown by the color scale.

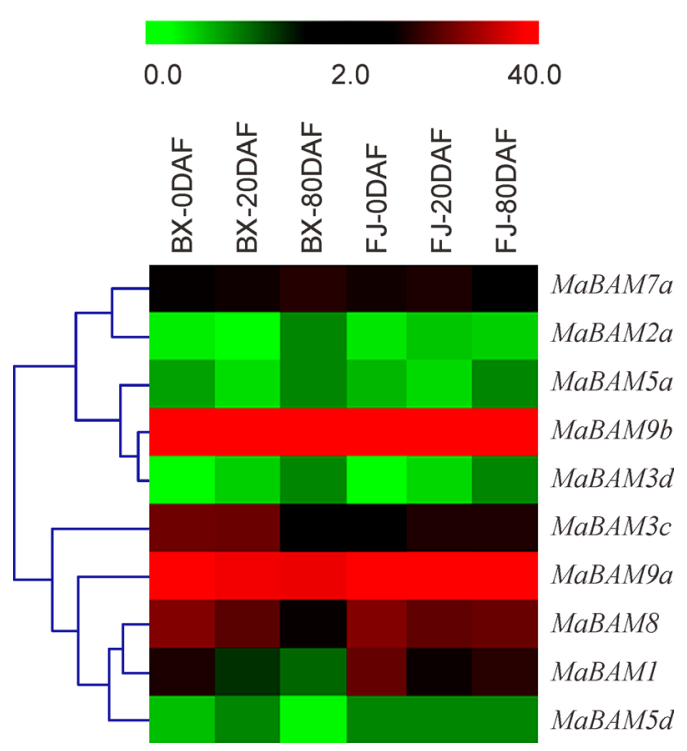

Fig. 5 Expression patterns of $M a B A M s$ at different stages of fruit development in two banana cultivars, BX and FJ cultivars. The heat map with clustering was created based on the FPKM of MaBAMs. Differences in gene expression changes are shown by the color scale.

For FJ, nine (no $M a B A M 5 d$ ), nine (no $M a B A M 5 d$ ), and seven (no MaBAM3d, MaBAM5a, and MaBAM5d) $M a B A M$ genes were expressed at 0,20 , and $80 \mathrm{DAF}$, among which four (MaBAM1, MaBAM8, MaBAM9a, and MaBAM9b), three (MaBAM8, MaBAM9a, and $M a B A M 9 b)$, and three (MaBAM8, MaBAM9a, and $M a B A M 9 b)$ genes, respectively, showed high transcript accumulation $(>10)$ at each stage. Notably, MaBAM8, $M a B A M 9 a$, and MaBAM9b displayed high transcripts levels $(>15)$ at all stages of fruit development.

Comparison of the expression patterns of banana $M a B A M$ genes at distinct phases of fruit development in both $\mathrm{BX}$ and FJ revealed that some MaBAM genes has similar high expression levels at 0 and 20 DAF in both BX and FJ, including MaBAM8, MaBAM9a, and MaBAM9b. This indicates that these genes have similar functions during early fruit developmental stages of both BX and FJ. However, the MaBAM3c had abundant transcripts $(>18)$ at $0 \mathrm{DAF}$ in $\mathrm{BX}$, but low transcripts $(<4)$ at $0 \mathrm{DAF}$ in FJ. In contrast, $M a B A M 1$ was highly expressed $(>16)$ at 0 DAF in FJ, but had low expression levels $(<8.2)$ in BX. This finding indicates that some MaBAM members have different transcriptional responses during early fruit development in both BX and FJ. In addition, MaBAM9a and $M a B A M 9 b$ displayed high transcriptional abundance $(>30)$ at all development phases in both BX and FJ, indicating key role for these two genes in regulating banana fruit development.

\subsection{Expression profiles of $M a B A M$ genes in different stages of fruit ripening in two banana cultivars}

To investigate the transcriptional response of $M a B A M$ genes in the banana fruit ripening process, the expression patterns of the MaBAM genes were measured in fruits sampled at 0,8 , and $14 \mathrm{DPH}$ in $\mathrm{BX}$, and 0,3 , and $6 \mathrm{DPH}$ in FJ (Fig. 6; Appendix A, Table S4). Among the 16 MaBAM genes, nine showed expression at different stages of banana fruit ripening.

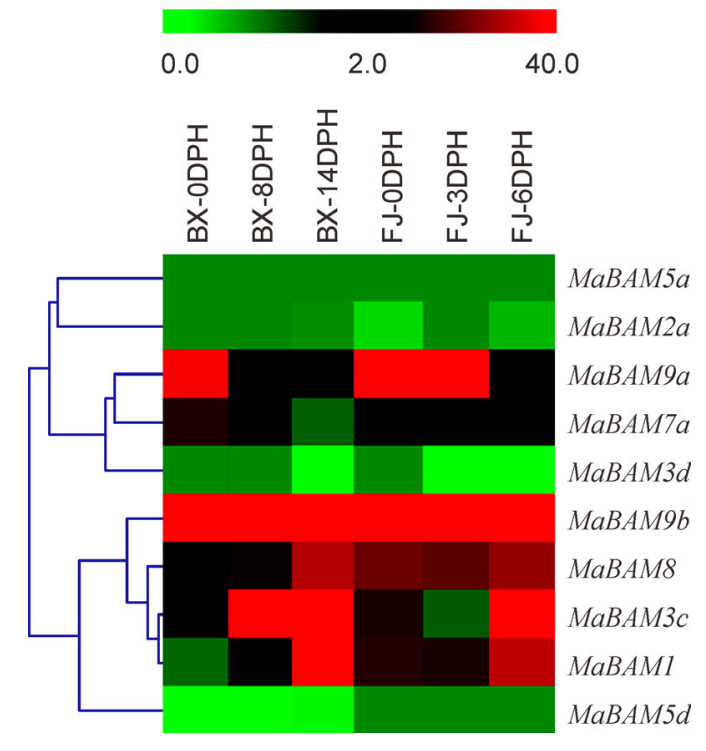

Fig. 6 Expression patterns of MaBAMs at different stages of fruit ripening in two banana cultivars, BX and FJ cultivars. The heat map with clustering was created based on the FPKM of MaBAMs. Differences in gene expression changes are shown by the color scale. 
For BX, seven (no MaBAM2a and MaBAM3d), seven (no $M a B A M 2 a$ and $M a B A M 3 a$ ), and nine $M a B A M$ genes were expressed at 0,8 , and $14 \mathrm{DPH}$, respectively, of which two (MaBAM9a and MaBAM9b), two (MaBAM3c and $M a B A M 9 b)$, and four (MaBAM1, MaBAM3c, MaBAM8, and $M a B A M 9 b)$ genes, respectively, showed high expression levels $(>25)$ at each stage. Additionally, MaBAM9b exhibited high transcript levels $(>640)$ at all stages of fruit ripening.

For FJ, seven (no MaBAM3d and MaBAM5d), seven (no $M a B A M 2 a$ and MaBAM5d), and eight (no MaBAM5d) $M a B A M$ genes were expressed at 0,3 , and $6 \mathrm{DPH}$, respectively, of which three (MaBAM8, MaBAM9a, and $M a B A M 9 b)$, three $(M a B A M 8, M a B A M 9 a$, and $M a B A M 9 b)$, and four (MaBAM1, MaBAM3c, MaBAM8, and $M a B A M 9 b)$ genes, respectively, showed high transcript accumulation $(>15)$ at each stage. Additionally, $M a B A M 8$ and MaBAM $9 b$ exhibited high expression levels $(>15)$ at all stages of fruit ripening.

Comparison of the expression profiles of MaBAMs at different stages of fruit ripening indicated that four genes (MaBAM1, MaBAM3c, MaBAM8, and MaBAM9a) were expressed at all stages tested in both BX and FJ. Similarly, high expression $(>10)$ patterns were observed at late fruit ripening stages (14 DPH in BX and $6 \mathrm{DPH}$ in FJ) compared with the other stages, indicating that four $M a B A M$ genes had important roles in the late fruit ripening stage in both BX and FJ. However, the MaBAM 8 had abundant transcripts $(>15)$ at 0 and 3 DPH in FJ, but low transcripts $(<7.0)$ at 0 and $8 \mathrm{DPH}$ in BX. The MaBAM9a showed high expression levels $(>40)$ at $3 \mathrm{DPH}$ in FJ, yet low expression levels $(<4.0)$ were detected at $8 \mathrm{DPH}$ in $\mathrm{BX}$. In contrast, the MaBAM3c had high expression levels $(>280)$ at $8 \mathrm{DPH}$ in $\mathrm{BX}$, but low expression levels $(<1.5)$ at $3 \mathrm{DPH}$ in FJ. This finding indicates that some MaBAM members had different transcriptional responses during the fruit ripening processes in BX and FJ. In addition, $M a B A M 9 b$ displayed high transcriptional abundance $(>280)$ at all ripening phases in $\mathrm{BX}$ and $\mathrm{FJ}$, indicating a key role for this gene in regulating banana fruit ripening.

3.6 Expression profiles of $M a B A M$ genes in response to cold, salt, and osmotic stresses in two banana cultivars

Considerable evidence has indicated that $B A M$ genes participate in plant response to various abiotic stresses, including cold, salt, and drought. To better understand the role of MaBAM genes in response to these 3 stresses, their expression in leaves of BX and FJ was examined under cold, salt, and osmotic treatments using data from RNAseq assays (Fig. 7; Appendix A, Table S5). A total of 11 $M a B A M$ genes showed transcriptional changes after abiotic stress in BX and FJ.

For BX, eight (no MaBAM2a, MaBAM5a, and $M a B A M 5 c) M a B A M$ genes were upregulated $(>2)$ in response to cold, salt, and osmotic treatments, respectively,

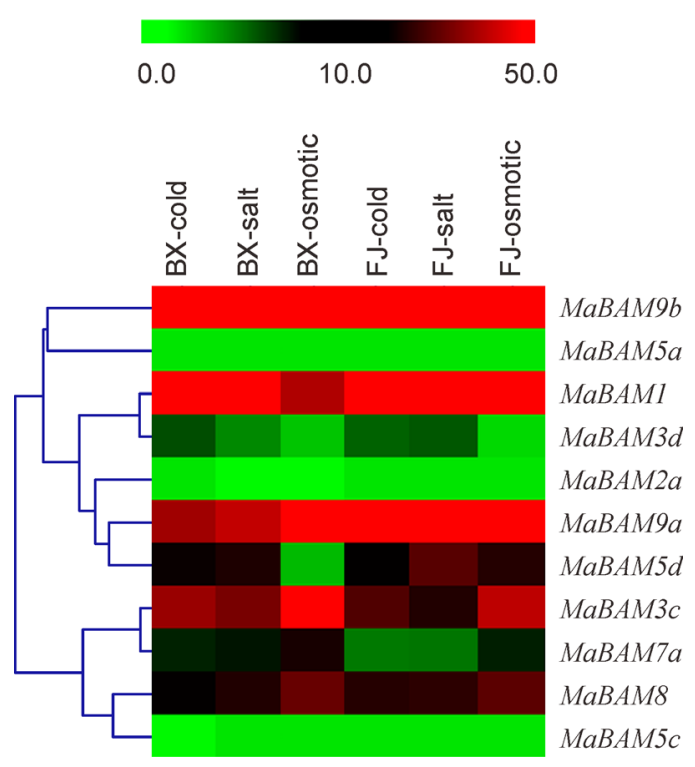

Fig. 7 Expression patterns of MaBAMs in response to cold, salt, and osmotic stresses in two banana cultivars, BX and FJ cultivars. The heat map with clustering was created based on the FPKM of MaBAMs. Differences in gene expression changes are shown by the color scale.

whereas the MaBAM5c, MaBAM2a, and MaBAM2a genes were downregulated $(<0.5)$ under cold, salt, and osmotic treatment, respectively. Additionally, five genes (MaBAM1, MaBAM3c, MaBAM8, MaBAM9a, and $M a B A M 9 b)$ were strongly upregulated $(>10)$ after each of the stress treatments.

For FJ, eight (no MaBAM2a, MaBAM5a, and $M a B A M 5 c$ ), eight (no MaBAM2a, MaBAM5a, and $M a B A M 5 c$ ), and seven (no MaBAM2a, MaBAM3d, $M a B A M 5 a$, and MaBAM5c) MaBAM genes were upregulated in response to cold, salt, and osmotic stress, respectively, whereas the MaBAM3d gene was downregulated under osmotic treatment. Additionally, six genes (MaBAM1, MaBAM3c, MaBAM5d, MaBAM8, MaBAM9a, and $M a B A M 9 b)$ were strongly upregulated $(>10)$ under all three stresses.

From these results, five genes (MaBAM1, MaBAM3c, $M a B A M 8, M a B A M 9 a$, and MaBAM9b) were strongly induced by the three stress treatments, indicating that they have similar function in BX and FJ under cold, salt, and osmotic stress. However, two genes (MaBAM5d and $M a B A M 7 a$ ) showing differential expression patterns between BX and FJ under osmotic treatment. This finding indicates that some MaBAM members have different transcriptional responses to osmotic stress treatment in $\mathrm{BX}$ and FJ.

3.7 Hormone-related and stress-related cis-acting elements in the promoters of $M a B A M$ genes

A promoter is a molecular switch that initiates gene 
Table 1 Kinds and numbers of known hormone-related and stress-related elements in the upstream regions of MaBAM genes

\begin{tabular}{|c|c|c|c|c|c|c|c|c|c|c|c|c|c|c|}
\hline Element & $\begin{array}{l}\text { ABRE } \\
\text { (ABA) }\end{array}$ & $\begin{array}{c}\text { ARE } \\
\text { (Anaerobic) }\end{array}$ & $\begin{array}{l}\text { AuxRR } \\
\text { (Auxin) }\end{array}$ & $\begin{array}{l}\text { Box-W1 } \\
\text { (Fungal) }\end{array}$ & $\begin{array}{c}\text { CGTCA- } \\
\text { motif } \\
\text { (MeJA) }\end{array}$ & Circadian & $\begin{array}{c}\text { ERE } \\
\text { (Ethylene) }\end{array}$ & $\begin{array}{c}\text { GARE } \\
\text { (Gibberellin) }\end{array}$ & $\begin{array}{l}\text { HSE } \\
\text { (Heat) }\end{array}$ & $\begin{array}{c}\text { LTR } \\
\text { (Cold) }\end{array}$ & $\begin{array}{c}\text { MBS } \\
\text { (Drought) }\end{array}$ & $\begin{array}{l}\text { TCA- } \\
\text { element } \\
\text { (Salicylic } \\
\text { acid) }\end{array}$ & $\begin{array}{l}\text { TC- } \\
\text { rich } \\
\text { repeats } \\
\text { (Defense) }\end{array}$ & Total \\
\hline MaBAM1 & 3 & 1 & 1 & 1 & 3 & & & 2 & & & & 1 & & 12 \\
\hline MaBAM2a & & 2 & & & & & & 1 & 3 & & & 1 & 1 & 8 \\
\hline MaBAM2b & & 2 & & & & & & 1 & 3 & & & 1 & 1 & 8 \\
\hline МaBAM3a & & & & 1 & 2 & 1 & & 1 & & & 2 & 1 & 2 & 10 \\
\hline MaBAM3b & & 1 & & & & 1 & & & & & 1 & 1 & 1 & 5 \\
\hline MaBAM3c & 1 & 2 & 1 & & 1 & 1 & 1 & & 1 & & 1 & & & 9 \\
\hline MaBAM3d & 2 & 1 & & & 3 & & & 1 & & 1 & 1 & & & 9 \\
\hline MaBAM5a & 2 & 1 & & & 6 & 1 & & 1 & & & & & 1 & 12 \\
\hline MaBAM5b & 2 & 1 & & & 6 & 1 & & 2 & & & & & & 12 \\
\hline MaBAM5c & 2 & 1 & & & 6 & 1 & & 2 & & & & & & 12 \\
\hline MaBAM5d & 3 & 3 & & & 4 & 1 & & 1 & & & & & 1 & 13 \\
\hline MaBAM7a & & 2 & & & & & & 1 & 3 & & & 1 & 1 & 8 \\
\hline MaBAM7b & & 2 & & & & & & 1 & 3 & & & 1 & 1 & 8 \\
\hline MaBAM8 & & & & 1 & 1 & 1 & & 1 & & & & & & 4 \\
\hline MaBAM9a & 1 & & 1 & 1 & 3 & 3 & & 1 & & 2 & & & & 12 \\
\hline MaBAM9b & 1 & 2 & & 1 & 2 & & & & & & 1 & & 1 & 8 \\
\hline
\end{tabular}

expression and studies of promoter cis-acting elements are very useful for investigating genes expression regulatory mechanism and potential functions ${ }^{[36]}$. BAM promoters have been documented as having roles in plant development and abiotic stresses in many species ${ }^{[5]}$. However, there are no such reports in banana. We chose banana MaBAMs for identification of potential promoter cis-acting elements to aid future understanding of the regulation of their expression and biological function. As shown in Table 1, five hormone-related (ABA, auxin, MeJA, ethylene, and gibberellin) and seven stress-related (anaerobic, fungal, heat, cold, drought, salicylic acid, and defense) elements were identified in the promoters of these $M a B A M$ genes. No less than four hormone- and stressrelated elements were found in the $16 B A M$ promoters, suggesting that BAMs participate in the regulation of development, ripening, and stress response by hormoneand stress-related cis-acting elements.

\section{Discussion}

Despite the economic and social importance of banana, compared to other crops, research on banana has evolved slowly. To improve banana fruit quality and enhance stress tolerance, it is essential to explore the mechanisms underlying banana fruit development, ripening, and response to abiotic stresses. Plant $B A M$ genes have various roles in plant growth, development and response to abiotic stresses, and are considered crucial regulators in multiple biological processes. However, limited information has been reported on the $M a B A M$ gene family of banana. In this study, we performed genome-wide identification and characterization of expression of the MaBAM gene family during development, ripening, and response to cold, salt, and osmotic stresses in banana.

4.1 Identification and evolutionary analysis of banana $M a B A M$ genes

In this study, a total of $16 M a B A M$ family genes were identified in the banana A genome (M. acuminata, $2 n=$ 22). This finding indicates that the banana $M a B A M$ gene family has expanded compared to Arabidopsis ${ }^{[5]}$ and rice $^{[16]}$. Based on phylogenetic analysis, these genes were classified into four subfamilies, which was consistent with the classification of those in the Arabidopsis and rice ${ }^{[5,16]}$. The phylogenetic results were also supported by the gene structure, which indicated that $M a B A M$ genes contain three to nine exons, and each subfamily shared a similar exonintron organization (Fig. 2), which was also found in Arabidopsis, rice, and barley ${ }^{[5,16,37]}$. However, the number of exons in banana $B A M$ genes is greater than in barley $(7)^{[37]}$ and sweet potato $(2)^{[38]}$, implying that the banana $M a B A M$ genes have changed greatly during evolution. Conserved motif analysis indicated that all MaBAMs have the typical glyco hydro 14 domains, and each subfamily shares similar motifs, further supporting the classification of MaBAMs. Together, the identification and classification of the banana $B A M$ gene family was supported by 
evolutionary, genetic structure, and conserved motif analyses.

4.2 Involvement of $B A M$ genes in fruit development, postharvest ripening, and abiotic stress response in banana

Banana fruit yield and quality are directly affected by fruit developmental processes. Thus, it is necessary to understand the mechanisms involved in banana fruit development. The BAM family has been reported to participate in the plant development of many species such as apple ${ }^{[39]}$, Arabidopsis ${ }^{[17]}$, barley ${ }^{[18]}$, and rye ${ }^{[40]}$. In apple, the amount of BAM protein was markedly greater during fruit development ${ }^{[39]}$. Two BAM genes in Arabidopsis regulate shoot development ${ }^{[17]}$. The expression of a distinct transcript, from the barley Bmyl gene, suggested its potential involvement in grain development ${ }^{[18]}$. In rye, expression of two $B A M$ genes changed significantly during seed development ${ }^{[40]}$. However, whether BAMs participate in fruit development of banana remains unclear. In this study, we found that most banana $B A M$ genes showed increased expression during fruit development. Moreover, many genes showed high transcriptional abundance $(>10)$ at all developmental stages of both BX and FJ (Fig. 5). Based on the evidence above, $B A M$ genes are likely to be involved in fruit development in banana.

Banana postharvest ripening is crucial to improving fruit quality and extending fruit shelf life ${ }^{[41,42]}$. In this study, the number of $B A M$ genes with high transcript abundance $(>15)$ at $14 \mathrm{DPH}$ in BX and $6 \mathrm{DPH}$ in FJ was greater than that at other stages (Fig. 6), suggesting that $B A M$ genes are crucial in the late stages of banana fruit ripening. Previous evidence from other fruits has revealed the importance of $B A M$ genes during the late stages of ripening ${ }^{18,43,44]}$. In tomato, $B A M$ gene transcript accumulation and enzyme activity were elevated during the late stages of fruit ripening and had an important role in starch depletion and ripening process ${ }^{[43]}$. The barley Bmy2 protein was increased markedly in mature grain and decreased in developing grain ${ }^{[18]}$. In mango, BAM protein was involved in starch degradation during fruit ripening ${ }^{[4]}$. Overall, these results suggest the importance of $B A M$ genes in regulating fruit ripening.

Increasing evidence has suggested that $B A M$ genes could transcriptionally respond to multiple abiotic stresses in various plant species ${ }^{[2,19-21,36]}$. Further biochemical and genetic evidence has demonstrated that plant BAM proteins, acting as signal regulators, positively regulate plants response to drought ${ }^{[20]}$, cold $^{[22]}$, and salt stresses ${ }^{[23]}$. In this study, many $B A M$ genes showed significant changes after cold, salt, and osmotic treatment in the two banana cultivars, suggesting their potential role in the banana abiotic stress responses. Comparison of the expression patterns of $B A M s$ in $\mathrm{BX}$ and $\mathrm{FJ}$ under abiotic stress showed that $B A M$ genes similar expression patterns (Fig. 7), indicating that they function similarly in $\mathrm{BX}$ and $\mathrm{FJ}$ under the cold, salt, and osmotic treatments. Collectively, it is concluded that $B A M$ genes are potentially important in contributing to abiotic stress responses in two banana cultivars.

\subsection{Cis-elements analysis in the promoters of $B A M$ genes}

It has been reported that BAM promoters participate in multiple developmental processes and abiotic stress responses in many species. The Arabidopsis Ct-Bmy promoter is involved in the development of chloroplastlocalized transitory leaf $\operatorname{starch}^{[45]}$. Expression of two Arabidopsis BAM promoters in leaves, sepals, siliques or root tips suggested their potential control of starch breakdown in different tissues ${ }^{[19]}$. In sweet potato, activity of BAM promoter was exclusively detected in the sugar metabolism process ${ }^{[46]}$. In Poncirus trifoliata, BAM1 contains a CBF-recognizing element and is important in cold tolerance by modulating soluble sugar levels ${ }^{[36]}$. Promoter activity analysis of Arabidopsis BAM1 suggested that thioredoxin-regulated BAM1 activates starch degradation in illuminated mesophyll cells under osmotic stress $^{[5]}$. Additionally, gibberellic acid- and abscisic acidresponsive elements in the promoter of $A M Y$ genes have been identified in barley ${ }^{[47]}$ and wheat ${ }^{[48]}$. In rice, the function of the AMY promoter is in the growth or development of mature leaves, stems, sheaths, roots, and seeds ${ }^{[49]}$. However, no study has reported hormone-related elements of the BAM promoter. In this study, five hormone-related and seven stress-relevant elements were analyzed in the promoters of $16 M a B A M$ genes (Table 1), indicating that BAMs participate in the regulation of multiple development process and stress responses by hormone-related and stress-related cis-acting elements. These findings have provided a solid foundation for further studies of the BAM protein-mediated development and abiotic stresses signal response in banana.

\section{Conclusions}

This study identified $16 B A M$ genes from the banana genome and established their classification and evolutionary relationship using phylogenetic, gene structure, and conserved protein motif analyses. Expression analyses demonstrated that banana $B A M$ genes are involved in regulating fruit development, the late stage of ripening, and abiotic stress responses. Furthermore, hormone-related and stress-related cis-acting elements analyses of MaBAM promoters indicated that they are potentially involved in responding to developmental, ripening, and abiotic stresses signaling. These results will advance the understanding of the functional characterization of $B A M$ genes in the banana developmental and ripening processes, and in responses to 
abiotic and biotic stresses, and provide a solid foundation for further genetic improvement of banana quality and resistance to various stresses.

Acknowledgements This work was supported by the National Natural Science Foundation of China (31401843), the Modern Agro-industry Technology Research System (CARS-32), the National Nonprofit Institute Research Grant of CATAS-ITBB (1630052016006), the National Program on Key Basic Research Project of China (2014CB160314), and the Natural Science Foundation of Hainan Province (314100).

Supplementary materials The online version of this article at http://dx. doi.org/10.15302/J-FASE-2016127 contains supplementary materials (Appendix A).

Compliance with ethics guidelines Hongxia Miao, Peiguang Sun, Yulu Miao, Juhua Liu, Jianbin Zhang, Caihong Jia, Jingyi Wang, Zhuo Wang, Zhiqiang Jin, and Biyu Xu declare that they have no conflict of interest or financial conflicts to disclose.

This article does not contain any studies with human or animal subjects performed by any of the authors.

\section{References}

1. Sethi S, Saini J S, Mohan A, Brar N K, Verma S, Sarao N K, Gill K S. Comparative and evolutionary analysis of $\alpha$-amylase gene across monocots and dicots. Functional \& Integrative Genomics, 2016, 16 (5): 545-555

2. Zanella M, Borghi G L, Pirone C, Thalmann M, Pazmino D, Costa A, Santelia D, Trost P, Sparla F. $\beta$-amylase 1 (BAM1) degrades transitory starch to sustain proline biosynthesis during drought stress. Journal of Experimental Botany, 2016, 67(6): 1819-1826

3. Smith A M, Zeeman S C, Smith S M. Starch degradation. Annual Review of Plant Biology, 2005, 56: 73-98

4. Zeeman S C, Delatte T, Messerli G, Umhang M, Stettler M, Mettler T, Streb S, Reinhold H, Kotting O. Starch breakdown: recent discoveries suggest distinct pathways and novel mechanisms. Functional Plant Biology, 2007, 34(6): 465-473

5. Valerio C, Costa A, Marri L, Issakidis-Bourguet E, Pupillo P, Trost $\mathrm{P}$, Sparla F. Thioredoxin-regulated $\beta$-amylase (BAM1) triggers diurnal starch degradation in guard cells, and in mesophyll cells under osmotic stress. Journal of Experimental Botany, 2011, 62(2): 545-555

6. Asatsuma S, Sawada C, Itoh K, Okito M, Kitajima A, Mitsui T. Involvement of $\alpha$-amylase I-1 in starch degradation in rice chloroplasts. Plant \& Cell Physiology, 2005, 46(6): 858-869

7. Ziegler P. Cereal $\beta$-amylases. Journal of Cereal Science, 1999, 29 (3): 195-204

8. Weise S E, Weber A P, Sharkey T D. Maltose is the major form of carbon exported from the chloroplast at night. Planta, 2004, 218(3): 474-482

9. Vester-Christensen M B, Hachem M A, Svensson B, Henriksen A. Crystal structure of an essential enzyme in seed starch degradation: barley limit dextrinase in complex with cyclodextrins. Journal of Molecular Biology, 2010, 403(5): 739-750

10. Sørensen A, Ahring B K, Lübeck M, Ubhayasekera W, Bruno K S, Culley D E, Lübeck P S. Identifying and characterizing the most significant $\beta$-glucosidase of the novel species Aspergillus. Canadian Journal of Microbiology, 2012, 58(9): 1035-1046

11. Mizanur R M, Griffin A K, Pohl N L. Recombinant production and biochemical characterization of a hyperthermostable $\alpha$-glucan/ maltodextrin phosphorylase from Pyrococcus furiosus. Archaea, 2008, 2(3): 169-176

12. Lloyd J R, Kossmann J, Ritte G. Leaf starch degradation comes out of the shadows. Trends in Plant Science, 2005, 10(3): 130-137

13. Yu T S, Zeeman S C, Thorneycroft D, Fulton D C, Dunstan H, Lue W L, Hegemann B, Tung S Y, Umemoto T, Chapple A, Tsai D L, Wang S M, Smith A M, Chen J, Smith S M. $\alpha$-Amylase is not required for breakdown of transitory starch in Arabidopsis leaves. Journal of Biological Chemistry, 2005, 280(11): 9773-9779

14. Fulton D C, Stettler M, Mettler T, Vaughan C K, Li J, Francisco P, Gil M, Reinhold H, Eicke S, Messerli G, Dorken G, Halliday K, Smith A M, Smith S M, Zeeman S C. $\beta$-AMYLASE4, a noncatalytic protein required for starch breakdown, acts upstream of three active $\beta$-amylases in Arabidopsis chloroplasts. Plant Cell, 2008, 20(4): $1040-1058$

15. Srivastava G, Kayastha A M. $\beta$-Amylase form starchless seeds of trigonella foenum-graecum and its localization in germinating seeds. PLoS One, 2014, 9(2): e88697

16. Mason-Gamer R J. The $\beta$-amylase genes of grasses and a phylogenetic analysis of the Triticeae (Poaceae). American Journal of Botany, 2005, 92(6): 1045-1058

17. Reinhold H, Soyk S, Simkova K, Hostettler C, Marafino J, Mainiero S, Vaughan C K, Monroe J D, Zeeman S C. $\beta$-amylase-like proteins function as transcription factors in Arabidopsis, controlling shoot growth and development. Plant Cell, 2011, 23(4): 1391-1403

18. Vinje M A, Willis D K, Duke S H, Henson C A. Differential expression of two $\beta$-amylase genes (Bmyl and Bmy2) in developing and mature barley grain. Planta, 2011, 233(5): 1001-1010

19. Francisco P, Li J, Smith S M. The gene encoding the catalytically inactive $\beta$-amylase BAM4 involved in starch breakdown in Arabidopsis leaves is expressed preferentially in vascular tissues in source and sink organs. Journal of Plant Physiology, 2010, 167 (11): 890-895

20. Prasch C M, Ott K V, Bauer H, Ache P, Hedrich R, Sonnewald U. $\beta$ amlyase 1 mutant Arabidopsis plants show improved drought tolerance due to reduced starch breakdown in guard cells. Journal of Experimental Botany, 2015, 66(19): 6059-6067

21. Monroe J D, Storm A R, Badley E M, Lehman M D, Platt S M, Saunders L K, Schmitz J M, Torres C E. $\beta$-amylase1 and $\beta$-amylase3 are plastidic starch hydrolases in Arabidopsis that seem to be adapted for different thermal, $\mathrm{pH}$, and stress conditions. Plant Physiology, 2014, 166(4): 1748-1763

22. Kaplan F, Guy C L. RNA interference of Arabidopsis $\beta$-amylase8 prevents maltose accumulation upon cold shock and increases sensitivity of PSII photochemical efficiency to freezing stress. Plant Journal, 2005, 44(5): 730-743

23. Ahmed I M, Cao F, Han Y, Nadira U A, Zhang G, Wu F. Differential changes in grain ultrastructure, amylase, protein and amino acid profiles between Tibetan wild and cultivated barleys under drought and salinity alone and combined stress. Food Chemistry, 2013, 141(3): 2743-2750

24. Bierhals J D, Lajolo F M, Cordenunsi B R, Oliveira do Nascimento J 
R. Activity, cloning, and expression of an isoamylase-type starchdebranching enzyme from banana fruit. Journal of Agricultural and Food Chemistry, 2004, 52(24): 7412-7418

25. D'Hont A, Denoeud F, Aury J M, Baurens F C, Carreel F, Garsmeur O, Noel B, Bocs S, Droc G, Rouard M, Da Silva C, Jabbari K, Cardi C, Poulain J, Souquet M, Labadie K, Jourda C, Lengellé J, RodierGoud M, Alberti A, Bernard M, Correa M, Ayyampalayam S, Mckain M R, Leebens-Mack J, Burgess D, Freeling M, Mbéguié-AMbéguié D, Chabannes M, Wicker T, Panaud O, Barbosa J, Hribova E, Heslop-Harrison P, Habas R, Rivallan R, Francois P, Poiron C, Kilian A, Burthia D, Jenny C, Bakry F, Brown S, Guignon V, Kema G, Dita M, Waalwijk C, Joseph S, Dievart A, Jaillon O, Leclercq J, Argout X, Lyons E, Almeida A,Jeridi M, Dolezel J, Roux N, Risterucci A M, Weissenbach J, Ruiz M, Glaszmann J C, Quétier F, Yahiaoui N, Wincker P. The banana (Musa acuminata) genome and the evolution of monocotyledonous plants. Nature, 2012, 488 (7410): 213-217

26. Ravi I, Uma S, Vaganan M M, Mustaffa M M. Phenotyping bananas for drought resistance. Frontiers in Physiology, 2013, 4(4): 9

27. Yan Y, Takáč T, Li X, Chen H, Wang Y, Xu E, Xie L, Su Z, Šamaj J, $\mathrm{Xu}$ C. Variable content and distribution of arabinogalactan proteins in banana (Musa spp.) under low temperature stress. Frontiers in Plant Science, 2004, 6: 353

28. Yang Q S, Gao J, He W D, Dou T X, Ding I J, Wu J H, Li C Y, Peng $\mathrm{X}$ X, Zhang S, Yi G J. Comparative transcriptomics analysis reveals difference of key gene expression between banana and plantain in response to cold stress. BMC Genomics, 2015, 16(1): 446

29. Hirayama T, Shinozaki K. Research on plant abiotic stress responses in the post-genome era: past, present and future. Plant Journal, 2010, 61(6): 1041-1052

30. Hu W, Wang L, Tie W, Yan Y, Ding Z, Liu J, Li M, Peng M, Xu B, Jin Z. Genome-wide analyses of the bZIP family reveal their involvement in the development, ripening and abiotic stress response in banana. Scientific Reports, 2016, 6: 30203

31. do Nascimento J R O, Júnior A V, Bassinello P Z, Cordenunsi B R, Mainardi J A, Purgatto E, Lajolo F M. $\beta$-amylase expression and starch degradation during banana ripening. Postharvest Biology and Technology, 2006, 40(1): 41-47

32. Pua E C, Chandramouli S, Han P, Liu P. Malate synthase gene expression during fruit ripening of Cavendish banana (Musa acuminata cv. Williams). Journal of Experimental Botany, 2003, 54(381): 309-316

33. Finn R D, Clements J, Eddy S R. HMMER web server: interactive sequence similarity searching. Nucleic Acids Research, 2011, 39 (suppl): 29-37

34. Tamura K, Peterson D, Peterson N, Stecher G, Nei M, Kumar S. MEGA5: molecular evolutionary genetics analysis using maximum likelihood, evolutionary distance, and maximum parsimony methods. Molecular Biology and Evolution, 2011, 28(10): 2731-2739

35. Trapnell C, Roberts A, Goff L, Pertea G, Kim D, Kelley D R, Pimentel H, Salzberg S L, Rinn J L, Pachter L. Differential gene and transcript expression analysis of RNA-seq experiments with TopHat and Cufflinks. Nature Protocols, 2012, 7(3): 562-578

36. Peng T, Zhu X, Duan N, Liu J H. PtrBAM1, a $\beta$-amylase-coding gene of Poncirus trifoliata, is a CBF regulon member with function in cold tolerance by modulating soluble sugar levels. Plant, Cell \& Environment, 2014, 37(12): 2754-2767

37. Yoshigi N, Okada Y, Sahara H, Tamaki T. A structural gene encoding $\beta$-amylase of barley. Bioscience, Biotechnology, and Biochemistry, 1995, 59(10): 1991-1993

38. Rajapakse S, Nilmalgoda S D, Molnar M, Ballard R E, Austin D F, Bohac J R. Phylogenetic relationships of the sweetpotato in Ipomoea series Batatas (Convolvulaceae) based on nuclear $\beta$-amylase gene sequences. Molecular Phylogenetics and Evolution, 2004, 30(3): 623-632

39. Zhang D, Wang Y. $\beta$-amylase in developing apple fruits: activities, amounts and subcellular localization. Science China. Life Sciences, 2002, 45(4): 429-440

40. Rorat T, Sadowski J, Irzykowski W, Ziegler P, Daussant J. Differential expression of two $\beta$-amylase genes of rye during seed development. Physiologia Plantarum, 1995, 94(1): 19-24

41. Shiga T M, Soares C A, Nascimento J R, Purgatto E, Lajolo F M, Cordenunsi B R. Ripening-associated changes in the amounts of starch and non-starch polysaccharides and their contributions to fruit softening in three banana cultivars. Journal of the Science of Food and Agriculture, 2011, 91(8): 1511-1516

42. Etienne A, Génard M, Bugaud C. A process-based model of TCA cycle functioning to analyze citrate accumulation in pre- and postharvest fruits. PLoS One, 2015, 10(6): e0126777

43. Maria T, Tsaniklidis G, Delis C, Nikolopoulou A E, Nikoloudakis N, Karapanos I, Aivalakis G. Gene transcript accumulation and enzyme activity of $\beta$-amylases suggest involvement in the starch depletion during the ripening of cherry tomatoes. Plant Gene, 2016, 5: $8-12$

44. Peroni F H, Koike C, Louro R P, Purgatto E, do Nascimento J R, Lajolo F M, Cordenunsi B R. Mango starch degradation. II. The binding of $\alpha$-amylase and $\beta$-amylase to the starch granule. Journal of Agricultural and Food Chemistry, 2008, 56(16): 7416-7421

45. Lao N T, Schoneveld O, Mould R M, Hibberd J M, Gray J C, Kavanagh T A. An Arabidopsis gene encoding a chloroplasttargeted $\beta$-amylase. Plant Journal, 1999, 20(5): 519-527

46. Maeo K, Tomiya T, Hayashi K, Akaike M, Morikami A, Ishiguro S, Nakamura K. Sugar-responsible elements in the promoter of a gene for $\beta$-amylase of sweet potato. Plant Molecular Biology, 2001, 46 (5): $627-637$

47. Gubler F, Jacobsen J V. Gibberellin-responsive elements in the promoter of a barley high-pI $\alpha$-amylase gene. Plant Cell, 1992, 4 (11): 1435-1441

48. Huttly A K, Phillips A L, Tregear J W. Localisation of cis elements in the promoter of a wheat $\alpha$-Amy2 gene. Plant Molecular Biology, 1992, 19(6): 903-911

49. Chan M T, Chang H H, Ho S L, Tong W F, Yu S M. Agrobacteriummediated production of transgenic rice plants expressing a chimeric $\alpha$-amylasepromoter/ $\beta$-glucuronidase gene. Plant Molecular Biology, 1993, 22(3): 491-506 